\title{
Infección protésica mamaria por Mycobacterium fortuitum en una paciente con lupus eritematoso sistémico
}

\author{
Diego Lizaso, Mercedes García, Ana Aguirre y Amadeo Esposto
}

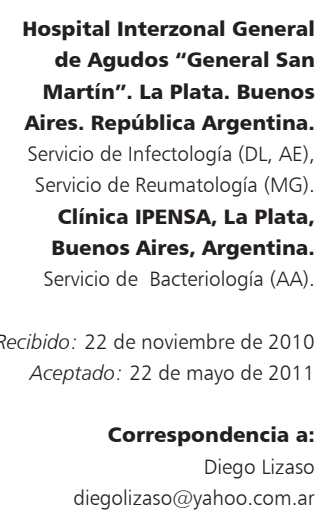

diegolizaso@yahoo.com.ar

\section{Breast implant infection by Mycobacterium fortuitum in a patient with systemic lupus erythematosus}

In recent decades there has been an increase in the number of breast implants for reconstruction and cosmetic purposes. Infection is a severe complication mostly caused by Staphylococcus aureus or coagulase-negative staphylococci. Mycobacteria are an infrequent cause of infection in this type of surgery. We describe a case of Mycobacterium fortuitum infection in a patient with lupus, subjected to a prosthetic replacement. These patients are more prone to unusual opportunistic infections. Treatment always requires both removal of prosthetic material and antibiotic therapy.

Key words: Breast implants; atypical mycobacterial infection; systemic lupus erythematosus; Mycobacterium fortuitum.

Palabras clave: Implantes mamarios, infecciones por micobacterias atípicas, Mycobacterium fortuitum.

\section{Caso clínico}

$\mathrm{P}$ aciente con 50 años de edad, con diagnóstico de lupus eritematoso sistémico (LES) hacía cuatro años. Durante su evolución presentó como intercurrencia un síndrome antifosfolipídico y nefritis lúpica que requirió tratamiento con anticoagulantes orales, azatioprina 100 mg/día, hidroxicloroquina 200 mg/día y prednisona 20 mg/día en dosis decreciente. En el año 1996 se le practicó una mastectomía bilateral por antecedentes familiares directos de neoplasia mamaria y hallazgos mamográficos de micro-calcificaciones agrupadas en ambas mamas. Con diagnóstico histopatológico de displasia severa se procedió a la colocación de prótesis mamarias texturizadas por vía submuscular. En agosto de 2008 fue sometida a un recambio protésico mamario bilateral, por encapsulamiento y retracción cutánea, colocándose prótesis texturizadas por vía retromuscular con incisión inframamaria. Al mes del procedimiento, comenzó con equivalentes febriles, tumefacción y eritema en ambas mamas. Se realizó una ecografía de tejidos blandos que demostró una colección periprotésica derecha evidente y mínima contralateral. Se le indicó reposo, hielo y anti-inflamatorios. Luego de una mejoría transitoria recrudeció el cuadro por lo que se le prescribió amoxicilina/ác. clavulánico (3 gr/ día) que luego fue modificado a ciprofloxacina asociada a clindamicina. Posteriormente, consultó a un médico infectólogo quien le aconsejó retiro de ambas prótesis mamarias dado el hallazgo ecográfico de colecciones bilaterales; sin embargo, la paciente no accedió. Evolucionó posteriormente con drenaje espontáneo de secreción en la mama derecha, por lo que se realizó una evacuación quirúrgica, obteniéndose un líquido de aspecto citrino que se envió a cultivo (Figuras 1 y 2).

Las muestras que se obtuvieron fueron procesadas de acuerdo al siguiente protocolo: baciloscopia mediante tinción de Ziehl Neelsen, siembra (directa con previa decontaminación por método de Petroff) en medios de Lowenstein Jensen y Stonebrink. La velocidad de desarrollo fue a los 5 días $\left(a 37^{\circ} \mathrm{C}\right)$; colonia con pigmentación: no cromógena; prueba de niacina negativa; reacción de catalasa positiva tanto a temperatura ambiente como a $68^{\circ} \mathrm{C}$; reducción de nitratos: positiva. Bajo la sospecha de micobacteria no tuberculosa de crecimiento rápido, se remitió la cepa a la Administración Nacional de Laboratorios e Institutos de Salud "Dr Carlos G. Malbrán”.

En el control de laboratorio presentaba los siguientes valores: hematocrito 32\%, hemoglobina: 10,6 gr\%, recuento de leucocitos $6.040 / \mathrm{mm}^{3}$ (neutrófilos: $71 \%$, linfocitos: 9,4\%), creatininemia $0,82 \mathrm{mg} \%$, uremia 0,3 $\mathrm{gr} / \mathrm{L}$, eritrosedimentación $42 \mathrm{~mm}$ en la primera hora, proteína C reactiva (PCR) $26 \mathrm{mg} / \mathrm{L}$.

La paciente consultó al Servicio de Infectología del Hospital Interzonal General de Agudos “Gral San Martín” donde se le indicó ciprofloxacina 750 mg cada 12 horas asociado a cotrimoxazol en dosis de $40 \mathrm{mg} / \mathrm{kg} /$ día de sulfa.

Posteriormente, el Instituto Malbrán completó la tipificación en medio de Dubos enriquecido, informando que se trataba de Mycobacterium fortuitum obteniendo una concentración inhibitoria mínima (CIM) y una concentración bactericida mínima (99,9\% de inhibición y muerte bacilar respectivamente) a fármacos alternativos (Tabla 1, muestra 1).

Con dicho informe se decidió continuar con igual 


\begin{tabular}{|c|c|c|c|c|c|}
\hline \multirow[t]{2}{*}{ Antimicrobiano } & \multirow{2}{*}{$\begin{array}{l}\text { Nivel sérico posible } \\
\text { de alcanzar }(\mu \mathrm{g} / \mathrm{mL})\end{array}$} & \multicolumn{2}{|c|}{ Muestra 1} & \multicolumn{2}{|c|}{ Muestra 2} \\
\hline & & $\mathbf{C I M}(\mu \mathrm{g} / \mathrm{mL})$ & Interpretación & $\mathbf{C I M}(\mu \mathrm{g} / \mathrm{mL})$ & Interpretación \\
\hline Claritromicina & $1,4-2,9$ & 128 & Resistente & $>256$ & Resistente \\
\hline Ciprofloxacina & $0,8-5,4$ & $<0,125$ & Sensible & 8 & Resistente \\
\hline Amikacina & $15-25$ & 2 & Sensible & 4 & Sensible \\
\hline Sulfametoxazol & $46-120$ & 32 & Sensible & 128 & Resistente \\
\hline Cefoxitina & $24-110$ & 64 & Sensible & 256 & Resistente \\
\hline Doxiciclina & $2-64$ & 16 & Sensible & 32 & Sensible \\
\hline Imipenem & $18-60$ & 4 & Sensible & $>64$ & Resistente \\
\hline Cefotaxima & $21-100$ & 256 & Resistente & 256 & Resistente \\
\hline
\end{tabular}

tratamiento y se sugirió la remoción de las prótesis a lo que la paciente nuevamente se negó. El cuadro se mantuvo estable, con franca involución de la tumefacción de la mama derecha y secreción intermitente en la mama contralateral.

\section{Evolución}

Dos meses después la paciente comenzó con registros subfebriles matutinos y a las 48 horas presentó fiebre $\left(38,5^{\circ} \mathrm{C}\right)$ asociada a leucopenia $\left(1.400\right.$ leucocitos $/ \mathrm{mm}^{3}$ sin polimorfonucleares), interpretándose como toxicidad debida a cotrimoxazol ya que su LES se encontraba inactivo. Se decidió su internación por neutropenia más fiebre y comenzó tratamiento empírico con piperacilina/ tazobactam. Se constató en la ecografía mamaria de control la presencia de escaso líquido a nivel de la mama derecha realizándose una nueva toma de muestra para cultivo de microorganismos habituales y micobacterias (Tabla 1, muestra 2). Se comenzó tratamiento con factores estimulantes de colonias granulocíticas, evolucionando favorablemente. Pudo ser dada de alta con ciprofloxacina más amikacina de acuerdo a la sensibilidad in vitro de las muestras previas. Transcurrido aproximadamente dos meses se produjo la exposición espontánea de la prótesis mamaria y se debió suspender la amikacina por hipoacusia, acúfenos y microhematuria. Ante esta situación la paciente accedió a realizarse la extracción de ambas prótesis. Continuó con ciprofloxacina durante tres meses más a pesar que, al recibir los resultados de sensibilidad, se demostró un incremento de la CIM de todos los antimicrobianos ensayados (Tabla 1). Evolucionó con notable mejoría clínica y de los parámetros biológicos, sin la presencia de colecciones en los controles ecográficos.

\section{Discusión}

Por cientos de años las mujeres intentaron mejorar su apariencia, alterando el volumen de sus senos, primero a través de la modificación de las vestimentas y luego por

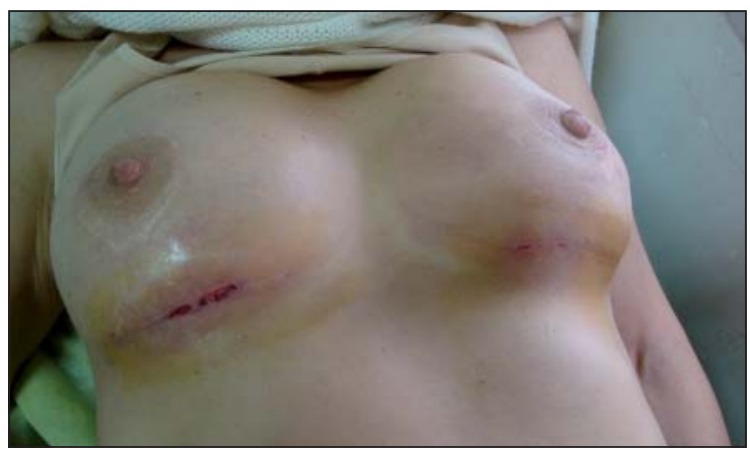

Figura 1. Mamas con signos de inflamación e incisión de drenaje de la mama derecha.

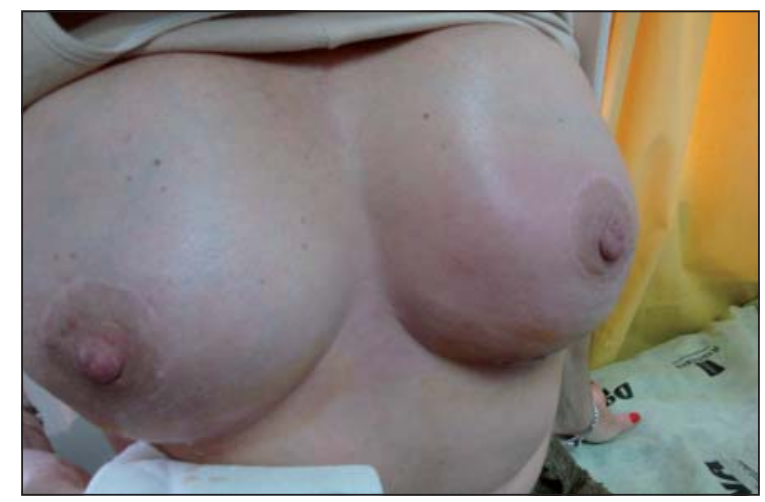

Figura 2. Mamas con signos de inflamación y escasa secreción serosa.

medio de la cirugía ${ }^{1}$. En 1895 se reportó por primera vez una reconstrucción mamaria exitosa, trasplantando un lipoma de cadera luego de remover un fibroadenoma mamario ${ }^{1}$; desde entonces se han utilizado múltiples técnicas y materiales con el propósito reconstructivo o estético.

En 1899, Gersuny introdujo el uso de inyecciones subcutáneas de parafina para lograr aumentar las dimensiones de las mamas ${ }^{1}$. Esta técnica se asoció a numerosas complicaciones como fístulas y úlceras luego de varios años del implante. 
En 1950 se comenzó a emplear silicona líquida, comprobándose con el tiempo la dispersión de la misma a través de la mama causando mastitis, destrucción del parénquima mamario, drenaje percutáneo de la silicona y migración abdominal del polímero ${ }^{1}$.

Las prótesis de gel de silicona fueron desarrolladas en 1963, presentando varias complicaciones durante sus primeros años de uso.

La mama no es una estructura estéril, contiene microbiota endógena derivada de los ductos del pezón, similar a la cutánea. Esta microbiota endógena puede ser responsable de la contaminación protésica en el tiempo del implante, particularmente cuando se utiliza una técnica trans-areolar.

La cirugía no está exenta de riesgos, Araco y cols, describen en 3.002 mujeres a las que se le colocaron implantes mamarios, una tasa de complicación de 4,6\%, siendo las más frecuente hematoma (1,5\%), seguido de infección (1,1\%), asimetría mamaria $(0,8 \%)$, ondulación $(0,7 \%)$ y retracción capsular $(0,5 \%)$. Al realizar el análisis multivariado encontró que la técnica usada al crear el bolsillo para colocar el implante (submuscular/biplano) se asoció en forma significativa con éstas² .

Dentro de las complicaciones, la infección es una de las principales, con una frecuencia aproximada de 2,5\%. Los riesgos se ven relacionados principalmente a la técnica quirúrgica y a la condición clínica del paciente.

Las infecciones agudas tienen una frecuencia que oscila entre 0 y $4 \%$ y ocurren, en su mayoría, durante el primer mes de colocado el implante ${ }^{1,3-5}$, presentándose como un cuadro febril con marcado eritema y dolor mamario. La ecografía puede ser de utilidad en demostrar una colección periprotésica. Mientras que las infecciones tardías ocurren meses o años luego de la cirugía y son relativamente infrecuentes $(2,8 \%)^{6,7}$.

La probabilidad de infección es alta cuando se realiza una reconstrucción y depende del tejido cicatricial preexistente y de la atrofia cutánea en el caso de radioterapia previa. Ambos sucesos se asocian a isquemia postoperatoria y al retraso en el cierre de la herida ${ }^{1,3,5}$.

Los pacientes oncológicos tienen más riesgo de infección protésica y ello está asociado a la radioterapia, el vaciamiento linfático, mastectomía subcutánea, colocación inmediata del implante, quimioterapia adyuvante e incisión periareolar. Por estas razones, hay un incremento de la incidencia en la cirugía reconstructiva ${ }^{1}$.

La incidencia de complicaciones perioperatorias en cirugías que requieren un expansor previo es de 5,8\%, siendo mayor luego de introducir el expansor (8,5\%) que de colocada la prótesis $(2,7 \%)^{5}$. La complicación más común es de tipo infeccioso (2,5\%) seguido de la necrosis del colgajo cutáneo $(2 \%)^{5}$.

Pajkos y cols, compararon los hallazgos bacteriológicos en la remoción de implantes en aquellas pacientes con y sin retracción capsular. Se detectaron cultivos positivos más frecuentemente en el primer grupo $(p=0,0006)$ y la mayoría de los microorganismos aislados fueron Staphylococcus coagulasa negativa, principalmente $S$. epidermidis. La microscopia electrónica confirmó la presencia de biopelícula en el implante y dentro de la cápsula ${ }^{8}$.

Actualmente hay más de 125 especies de micobacterias no tuberculosas (MNT) catalogadas ${ }^{9,10}$, ampliamente distribuidas en el medio ambiente, pudiéndose encontrar en el suelo y el agua. No hay evidencia de transmisión interhumana o del animal al hombre.

Las MNT que crecen en un tiempo igual o menor a 7 días son llamadas micobacterias de rápido crecimiento (MRC) (especialmente Mycobacterium chelonae, M. abscessus, y $M$. fortuitum) y su especie debe ser establecida por alguna metodología validada ${ }^{9,10}$.

Históricamente el grupo $M$. fortuitum incluyó tres especies: $M$. fortuitum, $M$. peregrinum y un tercer complejo sin nombre. Recientemente se han agregado nuevas especies como M. houstonense, M. boenickei y otras. Algunas especies ya establecidas han sido incluidas en este grupo como M. mageritense y $M$. senegalense. La identificación de éstas sólo puede ser realizar por métodos moleculares ${ }^{9}$.

Mycobacterium fortuitum puede afectar al pulmón (de manera similar a $M$. abscessus), piel, hueso y tejidos blandos, donde este agente es el más importante dentro de las MRC. Uno de los mecanismos de patogenicidad principales de esta especie es la capacidad de desarrollar biopelícula ${ }^{10}$.

Las infecciones por micobacterias atípicas en el campo de la estética han sido reportadas frecuentemente. En los tejidos blandos son más frecuentes las infecciones causadas por MRC (ejemplo M. fortuitum, M. chelonae, y M. abscessus). Pueden ser secundarias a mesoterapia ${ }^{11}$, liposucción ${ }^{12}$ o colocación de piercing ${ }^{13}$, siendo característico el inicio tardío entre 2 y 14 semanas de efectuado el procedimiento. Se manifiestan como absceso, reacción inflamatoria crónica y formación de fístulas. Las micobacterias más frecuentemente aisladas son $M$. abscessus y $M$. fortuitum ${ }^{4,11}$.

Se ha identificado al lavado de pies en lugares comunitarios durante un proceso de pedicuría, como un sitio de brote de forunculosis asociada a $M$. fortuitum ${ }^{14,15}$.

Las características clínicas de la infección por $M$. fortuitum posterior a la colocación de un implante mamario incluyen: eritema local, induración, molestia y presencia de líquido periprotésico. Los síntomas sistémicos como fiebre, calofríos o signos de sepsis generalmente no están presentes. La secreción por la herida frecuentemente es serosa o seropurulenta y sin olor ${ }^{4,7,16}$. Sin embargo, no hay características clínicas que permitan distinguirla de otras etiologías infecciosas subagudas o crónicas de baja virulencia, como Sthaphylococcus coagulasa negativa. El diagnóstico debe sospecharse en aquellos pacientes con 
la presentación clínica descrita que no responden a un tratamiento antimicrobiano empírico estándar.

El reconocimiento de estas infecciones inusuales es fundamental, debido a que el diagnóstico generalmente se retrasa, exponiéndose al paciente a un régimen antimicrobiano inadecuado, con la morbilidad y toxicidad que esto implica ${ }^{7}$. Macadam y cols, encuentran $M$. fortuitum en $73 \%$ en su serie de casos. Sin embargo, esta etiología puede ser sub-diagnosticada debido a que el cultivo para micobacterias es infrecuentemente realizado, por lo que una proporción de las infecciones con cultivos negativos podrían ser originadas por estos agentes ${ }^{4}$. El referido autor propone como probables mecanismos patogénicos la contaminación del sistema de agua en el momento del baño o que el microorganismo colonice la piel y no sea eliminado con la preparación pre-operatoria ${ }^{4}$.

Los pacientes con LES son susceptibles de padecer diversas infecciones oportunistas. El reporte más importante sobre MNT de la literatura médica en este tipo de pacientes incluye sólo 11 casos. El sitio de manifestación más frecuente fue la piel y los tejidos blandos. Al comparar micobacterias atípicas con micobacterias tuberculosas, encontraron que las primeras se manifiestan más frecuentemente en sitios extra-pulmonares $(p=0,006)$, se presentan en pacientes con mayor tiempo del diagnóstico de LES ( $p<0,001)$, ocurren en aquellos más añosos $(\mathrm{p}<0,001)$ y con mayor dosis acumulada de prednisona $(p=0,005)$. En el análisis de regresión logística se encontró únicamente al tiempo de evolución del LES como predictor de infección por $\mathrm{MNT}^{17}$.

El único reporte en Argentina de una paciente lúpica con infección por $M$. fortuitum, está referido a una paciente de 20 años, en tratamiento corticoesteroideo que evolucionó con múltiples bursitis purulentas, a lo que se le agrega posteriormente múltiples nódulos cutáneos de los que se aislaron $M$. fortuitum, evolucionando finalmente al óbito ${ }^{18}$.

El tratamiento inicial de cualquier infección postoperatoria protésica de mama incluye toma de cultivos y cobertura antimicrobiana empírica. Si no mejoran los síntomas y se sospecha de una infección periprotésica, está recomendado la remoción del material con desbridamiento quirúrgico del espacio periprotésico. Si el paciente presenta buena evolución y no desarrolla síntomas sistémicos se puede continuar con igual antibioterapia. Si se sospecha infección por MNT, está recomendado iniciar tratamiento con amikacina más cefoxitina, hasta obtener el informe de susceptibilidad in vitro. Tanto la identificación de especies como el test de susceptibilidad están recomendados siempre para un óptimo manejo ${ }^{4,7}$. El CLSI (Clinical and Laboratory Standards Institute) recomienda realizar el estudio de susceptibilidad in vitro por microdilución en caldo para el grupo de $M$. fortuitum $^{19}$. Los agentes antimicrobianos recomendados para testear las MRC son amikacina, cefoxitina, ciprofloxacina, claritromicina, doxiciclina, y sulfametoxazol (con o sin trimetoprim), así como también imipenem (sólo contra $M$. fortuitum) y tobramicina (sólo para M. chelonae $)^{19}$. Este estudio in vitro puede realizarse además por E-test ${ }^{\circledR}$.

La terapia adyuvante antimicrobiana es importante, debido a que los pacientes tratados sólo con cirugía, a menudo recaen a las cuatro a seis semanas ${ }^{20}$. Una vez que el informe de sensibilidad está disponible, la combinación de dos o más agentes antimicrobianos está recomendada para evitar el desarrollo de resistenciaa ${ }^{16,20,21}$. El tiempo óptimo de tratamiento sigue todavía sin resolverse, pero la experiencia sugiere que al menos seis meses de tratamiento son requeridos para la cura clínica y la reimplantación de la prótesis no debe realizarse hasta luego de cumplido este plazo ${ }^{4,7,20,21}$.

Mycobacterium fortuitum es generalmente susceptible a múltiples antimicrobianos, incluyendo amikacina (100\%), ciprofloxacina y ofloxacina (100\%), sulfonamidas (100\%), cefoxitina (50\%), imipenem (100\%), claritromicina (80\%) y doxiciclina $(50 \%)^{9}$.

El tratamiento de este agente es un problema muy importante debido a que la monoterapia para infecciones graves debe ser evitada por la posibilidad de desarrollar resistencia a través de mutaciones o de mecanismos inducibles (p.ej.: metilasas o bombas de eflujo) ${ }^{9,10,22,23}$. La presencia de la metilasa erm ha ido descrita en varias especies de micobacterias, aunque su presencia no siempre se correlaciona con una resistencia fenotípica ${ }^{22}$. Este fenómeno puede ser atribuido a la presencia de enzimas inducibles y la resistencia a macrólidos aparecer luego de la inducción con este antimicrobiano ${ }^{24}$.

La terapia combinada usando un macrólido (claritromicina tiene mejor actividad contra M. abscessus o M. chelonae $)^{9,25}$ o quinolonas (especialmente para $M$. fortuitum $)^{9,25}$ y un antimicrobiano parenteral (amikacina) mostró una actividad uniforme contra estos microorganismos, siendo la acción de cefoxitina e imipenem menos predecible $e^{9,25}$, pero recomendada para las infecciones graves.

El segundo problema importante es la presencia de un material extraño u otra condición clínica (p.ej.: paciente con fibrosis quística) que implica el desarrollo de biopelícula; su trascendencia es extrema en el manejo, debido a que presentan una mayor resistencia que la microbiota planctónica $^{10}$.

Para las infecciones graves de piel y tejidos blandos, el tratamiento debe ser al menos con dos agentes con actividad in vitro contra el aislado clínico y mantenido por cuatro meses. Generalmente está indicada la cirugía cuando hay una enfermedad extensa, absceso o el tratamiento antimicrobiano es dificultoso. La remoción de un cuerpo extraño como un implante de mama o un catéter es esencial para la recuperación ${ }^{9,10}$. 


\section{Conclusión}

Todas las pacientes que sean sometidas a un implante mamario pueden adquirir una infección asociada a la prótesis. Dentro de las etiologías, las micobacterias son una causa muy infrecuente y este reporte es, en nuestro conocimiento, la primera descripción de una infección protésica mamaria por $M$. fortuitum en una paciente lúpica. Esta etiología tienen por lo general menor traducción clínica y los cultivos de rutina son negativos, por lo que se necesita un alto índice de sospecha para su diagnóstico. Por esto, al presentarse una infección protésica, debe solicitarse tinción para microorganismos ácido-alcohol resistentes y cultivo para micobacterias. El tratamiento antimicrobiano combinado y el desbridamiento quirúrgico del implante es fundamental para una favorable evolución. La negativa de la paciente de acceder en reiteradas ocasiones a la extirpación de los implantes provocó un retraso notable en su curación, la inducción de resistencia a todos los antimicrobianos ensayados y nefrotoxicidad asociada a amikacina.

\section{Resumen}

En las últimas décadas se ha producido un incremento en el número de colocaciones de implantes mamarios para reconstrucciones y fines estéticos. La infección es una complicación seria y en su mayoría es producida por Staphylococcus aureus o Staphylococcus coagulasanegativa. Las micobacterias son una causa infrecuente de infección en este tipo de cirugías. Describimos el caso de una infección de un implante mamario por Mycobacterium fortuitum en una paciente lúpica sometida a un recambio protésico. Este tipo de pacientes es más propenso a padecer infecciones oportunistas. El tratamiento siempre requiere retiro del material asociado a antibioterapia.

\section{Referencias}

1.- Pittet B, Montandon D, Pittet D. Infection in breast implants. Lancet Infect Dis 2005; 5 (2): 94-106.

2.- Araco A, Gravante G, Araco F, Delogu D, Cervelli V, Walgenbach $\mathrm{K}$. A retrospective analysis of 3,000 primary aesthetic breast augmentations: postoperative complications and associated factors. Aesthetic Plast Surg 2007; 31 (5): 532-9.

3.- Brand K G. Infection of mammary prostheses: a survey and the question of prevention. Ann Plast Surg 1993; 30 (4): 289-95.

4.- Macadam S A, Mehling B M, Fanning A, Dufton J A, Kowalewska-Grochowska K T, Lennox P, et al. Nontuberculous mycobacterial breast implant infections. Plast Reconstr Surg 2007; 119 (1): 337-44.

5.- Cordeiro P G, McCarthy C M. A single surgeon's 12-year experience with tissue expander/implant breast reconstruction: part I. A prospective analysis of early complications. Plast Reconstr Surg 2006; 118 (4): 825-31.

6.- De Cholnoky T. Augmentation mammaplasty. Survey of complications in 10,941 patients by 265 surgeons. Plast Reconstr Surg 1970; 45 (6): 573-7.

7.- Vinh D C, Rendina A, Turner R, Embil J M. Breast implant infection with Mycobacterium fortuitum group: report of case and review. J Infect 2006; 52 (3): 63-7.

8.- Pajkos A, Deva A K, Vickery K, Cope C, Chang L, Cossart Y E. Detection of subclinical infection in significant breast implant capsules. Plast Reconstr Surg 2003; 111 (5): 1605-11.

9.- Griffith D E, Aksamit T, Brown-Elliott B A, Catanzaro A, Daley C, Gordin F, et al. An official ATS/IDSA statement: diagnosis, treatment, and prevention of nontuberculous mycobacterial diseases. Am J Respir Crit Care Med 2007 15; 175 (4): 367-416.

10.- Esteban J, Ortiz-Pérez A. Current treatment of atypical mycobacteriosis. Expert Opin Pharmacother 2009; 10 (17): 2787-99.

11.- Rivera-Olivero IA, Guevara A, Escalona A, Oliver M, Pérez-Alfonzo R, Piquero J, et al. Soft-tissue infections due to non-tuberculous mycobacteria following mesotherapy. What is the price of beauty. Enferm Infecc Microbiol Clin 2006; 24 (5): 302-6.

12.- Murillo J, Torres J, Bofill L, Ríos-Fabra A, Irausquin E, Isturiz R, et al. Skin and wound infection by rapidly growing mycobacteria: an unexpected complication of liposuction and liposculpture. The Venezuelan Collaborative Infectious and Tropical Diseases Study Group. Arch Dermatol 2000; 136 (11): 1347-52.

13.- Lewis C G, Wells M K, Jennings W C. Mycobacterium fortuitum breast infection following nipple-piercing, mimicking carcinoma. Breast J 2004; 10 (4): 363-5.

14.- Winthrop K L, Abrams M, Yakrus M, Schwartz I, Ely J, Gillies D, et al. An outbreak of mycobacterial furunculosis associated with footbaths at a nail salon. N Engl J Med 2002; 346 (18): 1366-71.

15.- Winthrop K L, Albridge K, South D, Albrecht P, Abrams M, Samuel M C, et al. The clinical management and outcome of nail salon-acquired Mycobacterium fortuitum skin infection. Clin Infect Dis 2004; 38 (1): 38-44.

16.- Haiavy J, Tobin H. Mycobacterium fortuitum infection in prosthetic breast implants. Plast Reconstr Surg 2002; 109 (6): 2124-8.

17.- Mok M Y, Wong S S, Chan T M, Fong D Y, Wong W S, Lau C S. Non-tuberculous mycobacterial infection in patients with systemic lupus erythematosus. Rheumatology
(Oxford) 2007; 46 (2): 280-4

18.- Laborde H, Rodrigue S, Catoggio P M. Mycobacterium fortuitum in systemic lupus erythematosus. Clin Exp Rheumatol 1989; 7 (3): 291-3.

19.- Woods G L. Susceptibility testing for mycobacteria. Clin Infect Dis 2000; 31 (5): 1209-15.

20.- Brown-Elliott B A, Wallace R J, Jr. Clinical and taxonomic status of pathogenic nonpigmented or late-pigmenting rapidly growing mycobacteria. Clin Microbiol Rev 2002; 15 (4): 716-46.

21.- Heistein J B, Mangino J E, Ruberg R L, Bergese J J. A prosthetic breast implant infected with Mycobacterium fortuitum. Ann Plast Surg 2000; 44 (3): 330-3.

22.- Esteban J, Martín-de-Hijas N Z, García-Almeida D, Bodas-Sánchez A, Gadea I, FernándezRoblas R. Prevalence of erm methylase genes in clinical isolates of non-pigmented, rapidly growing mycobacteria. Clin Microbiol Infect 2009; 15 (10): 919-23.

23.- Esteban J, Martín-de-Hijas N Z, Ortiz A, Kinnari TJ, Bodas Sánchez A, Gadea I, et al. Detection of lfrA and tap efflux pump genes among clinical isolates of non-pigmented rapidly growing mycobacteria. Int J Antimicrob Agents 2009; 34 (5): 454-6.

24.- Nash KA, Andini N, Zhang Y, Brown-Elliott BA, Wallace RJ, Jr. Intrinsic macrolide resistance in rapidly growing mycobacteria. Antimicrob Agents Chemother 2006; 50 (10): 3476-8.

25.- Fernández-Roblas R, Adames H, Martín-deHijas N Z, Almeida D G, Gadea I, Esteban J. In vitro activity of tigecycline and 10 other antimicrobials against clinical isolates of the genus Corynebacterium. Int J Antimicrob Agents 2009; 33 (5): 453-5. 